\title{
Primary Biliary Cholangitis Overlap With Autoimmune Hepatitis? A Case Report
}

\author{
Vitor Costa ${ }^{\mathrm{a}, \mathrm{c}}$, Marta Moreira ${ }^{\mathrm{b}}$, Raquel Costa ${ }^{\mathrm{a}}$, Joana Fontes ${ }^{\mathrm{a}}$
}

\begin{abstract}
Primary biliary cholangitis (PBC) is a chronic, autoimmune cholestatic disease, characterized by inflammation of the small and mediumsized bile ducts, which can lead to cirrhosis. Two to nineteen percent of patients with $\mathrm{PBC}$ have been reported to have features that overlap with autoimmune hepatitis (AIH). We report a case of a 39-year-old man with changes in liver biochemistry, with 6 years of asymptomatic evolution, whose diagnostic investigation determined the diagnosis of $\mathrm{PBC}$, however with some features of AIH.
\end{abstract}

Keywords: Primary biliary cholangitis; Ursodeoxycholic acid; Antimitochondrial antibodies; Cirrhosis; Autoimmune hepatitis; Overlap syndrome

\section{Introduction}

The major autoimmune disorders of the liver are the autoimmune hepatitis $(\mathrm{AIH})$, primary biliary cholangitis $(\mathrm{PBC})$ and primary sclerosing cholangitis (PSC).

$\mathrm{PBC}$ is a rare, chronic autoimmune cholestatic disease, whose incidence is higher in women, with female/male ratio up to $10: 1[1,2]$. The incidence of disease is greater between the fourth and fifth decade of life [3]. The worldwide incidence rate per 100,000 population ranges from 0.33 to 0.58 and prevalence ranges from 1.91 to 40.2 [4].

Although the etiology still remains unclear, immunogenetic and environmental interactions seem to trigger an intense autoimmune response against biliary epithelial cells which can lead ultimately to cirrhosis [5]. Histologically, $\mathrm{PBC}$ is characterized by a chronic and non-suppurative inflammation, which sur-

Manuscript submitted November 22, 2020, accepted December 1, 2020

Published online February 8, 2021

a Department of Medicine, Unidade Local de Saude do Alto Minho, Largo Conde de Bertiandos, Ponte de Lima 4990-041, Portugal

bDepartment of Gastroenterology, Unidade Local de Saude do Alto Minho, Largo Conde de Bertiandos, Ponte de Lima 4990-041, Portugal

${ }^{\mathrm{c} C o r r e s p o n d i n g ~ A u t h o r: ~ V i t o r ~ C o s t a, ~ D e p a r t m e n t ~ o f ~ M e d i c i n e, ~ U n i d a d e ~ L o c a l ~}$ de Saude do, Alto Minho, Largo Conde de Bertiandos, Ponte de Lima 4990041, Portugal. Email: vitor.costa@ulsam.min-saude.pt

doi: https://doi.org/10.14740/jmc3623 rounds and destroys interlobular and septal bile ducts, making it possible to find these changes in early stages of disease [6].

Clinically, patients may present fatigue, pruritus, jaundice, xanthomas, osteoporosis and dyslipidemia [7]. The disease should be suspected in patients with persistent cholestatic abnormalities for more than 6 months, especially an elevated alkaline phosphatase (ALP), or symptoms including pruritus or fatigue, which are important for the recognition of disease at early stages and to prompt treatment initiation, because this may alter the outcome, slow the progression and improve the survival [8]. In view of analytical changes in liver enzymes, these must be investigated properly.

The diagnosis is made if two of the following three criteria are satisfied: antimitochondrial antibodies (AMAs) titer $>$ $1: 40$, ALP $>1.5$ times the upper limit of normal for $>24$ weeks or liver histology with nonsuppurative destructive cholangitis and interlobular bile duct destruction [7].

After the diagnosis of PBC, the early start of treatment is fundamental to prevent the end-stage liver disease and to relieve the symptoms. Ursodeoxycholic acid (UDCA) is the first line of the treatment of $\mathrm{PBC}$, with many studies demonstrating clinical efficacy [6]. UDCA is a natural occurring hydrophilic bile acid, which appears to have less hepatotoxic properties compared to endogenous hydrophobic bile acids. Thus, the main therapeutic action of UDCA is to replace these endogenous acids by competitive inhibition at level of terminal ileum, increasing its secretion and elimination from hepatocytes. Anti-inflammatory and immunomodulatory properties of UDCA are also described [9]. A second line therapy is obeticholic acid (OCA), but this drug must be reserved for patients with inadequate response to UDCA (combination with UDCA) or as monotherapy in those intolerant to UDCA [6].

Regarding liver autoimmune diseases, some patients present overlapping features between the different disorders (AIH, PBC and PSC), being classified as having an "overlapping syndrome". This variant occurs in 3-7\% of patients with autoimmune liver disease [10].

\section{Case Report}

A 39-year-old man, without known pathological history or usual medication, was referred to the internal medicine consultation by his family doctor for analytical changes of liver biochemistry, known since 2014. The patient reported no history of fever, joint pains, rash, fatigue, itching, respiratory, 
gastrointestinal or cardiac complaints. He denied alcohol consumption.

On physical examination, he was anicteric, without adenopathies, hepatomegaly/splenomegaly, without stigmas of chronic liver disease.

From the study carried out, he had normal complete blood count, sedimentation rate of $38 \mathrm{~mm} / \mathrm{h}$, normal thyroid function, total bilirubin $0.57 \mathrm{mg} / \mathrm{dL}(0.3-1.2 \mathrm{mg} / \mathrm{dL})$, moderate elevation of gamma-glutamyl transpeptidase (GGT) $431 \mathrm{IU} / \mathrm{L}$ (normal < 55 IU/L) and ALP $210 \mathrm{IU} / \mathrm{L}$ (normal 30 - $120 \mathrm{IU} / \mathrm{L}$ ), with elevation of aspartate transaminase (AST) $81 \mathrm{IU} / \mathrm{L}$ (normal 8 - $35 \mathrm{IU} / \mathrm{L}$ ) and alanine transaminase (ALT) $135 \mathrm{IU} / \mathrm{L}$ (normal 10 - $45 \mathrm{IU} / \mathrm{L}$ ), with albumin $4.3 \mathrm{~g} / \mathrm{dL}$ and normal coagulation study. Viral serologies with immunity to hepatitis $\mathrm{b}$ virus (HBV), and the hepatitis c virus (HCV) and human immunodeficiency virus (HIV) were negative. With slightly increased immunoglobulin G (IgG) 1,772 mg/dL (normal 700 - 1,600 mg/dL) and increased immune globulin M (IgM) 676 $\mathrm{mg} / \mathrm{dL}$ (normal $40-230 \mathrm{mg} / \mathrm{dL}$ ). Study of iron, ceruloplasmin/ urinary copper, alpha fetoprotein, alpha-1 antitrypsin and lipid profile was normal. From the immunological study, he had positive AMA (antiM2) and negative antinuclear antibodies (ANAs) and anti-smooth muscle antibodies (ASMAs).

He had an abdominal ultrasound scan that was normal, and also had a magnetic resonance cholangiopancreatography that excluded other causes of intra and extra hepatic cholestasis. Liver biopsy showed expansion of port spaces by abundant inflammatory infiltrate, consisting predominantly of lymphocytes and plasma cells, rare eosinophils, with the presence of interface hepatitis, peri-portal fibrosis, with no evidence of steatosis, deposition of abnormal pigments, megamitochondria or Mallory bodies, and no evidence of malignancy.

With positive AMA (antiM2) and analytical pattern of cholestasis, diagnosis of $\mathrm{PBC}$ was assumed, with treatment with UDCA ( $250 \mathrm{mg}$ by pill) ( 1 pill in the morning, 1 by afternoon and 2 at night, total dose of $14 \mathrm{mg} / \mathrm{kg} / \mathrm{day}$ ). The patient tolerated the UDCA treatment, denying adverse effects. Analytical reassessment after 3 months of treatment showed improvement of liver biochemistry: GGT 129 IU/L, ALP $106 \mathrm{IU} / \mathrm{L}$, AST $29 \mathrm{IU} / \mathrm{L}$ and ALT $42 \mathrm{IU} / \mathrm{L}$. After 6 months of UDCA treatment, the result showed GGT 114 IU/L, AP 103 IU/L, AST $25 \mathrm{IU} / \mathrm{L}$, ALT $29 \mathrm{IU} / \mathrm{L}$, total bilirubin $0.46 \mathrm{mg} / \mathrm{dL}$, IgM $534 \mathrm{mg} / \mathrm{dL}$ and IgG $1,606 \mathrm{mg} / \mathrm{dL}$.

We will keep the patient in life-long follow-up to monitor the response to treatment, and manage the symptoms if these arise, while being attentive to the risk of cirrhosis or hepatocellular carcinoma. With the liver histology suggestive of AIH, if in future there is a raise of AST or ALT, we will consider the diagnosis of overlap syndrome, and associate corticoid treatment.

\section{Discussion}

PBC is a rare but important life-long autoimmune cholestatic disease of liver, leading to cholestasis and progressive fibrosis, which, over the time, can culminate in an end-stage biliary cirrhosis. Although it is most prevalent in women and those over the age of 50, the disease is not exclusive to these patients. We know that male, younger age at onset $(<45)$ and advanced disease at presentation are predictors of poor outcome [11]. In past, almost all patients were diagnosed at an advanced stage with classical signs and symptoms and the prognosis of these cases was often poor, with a life expectancy of only few years, evolving to terminal liver hepatic disease [12]. There is very important suspect of the disease, because many patients may be asymptomatic.

In this case report, the patient was asymptomatic, with changes from liver biochemistry with 6 years of evolution. We want to report this clinical case to warn about the need for an adequate investigation of changes in liver enzymes, especially after excluding the presence of biliary obstruction, alcohol or hepatotoxic drugs consumption, and confirmation of negative viral serologies. Sometimes, physicians devalue minor changes in liver biochemistry, associating this change with toxic habits, such as alcohol consumption. In this case, the patient denied alcoholism. It is important to know the criteria for diagnosis of autoimmune liver diseases, because they should be part of differential diagnosis of liver diseases. We know that the early treatment is fundamental to changing the natural history of disease.

Regarding our investigation, it is questionable to perform a liver biopsy in this patient, because we have two criteria to assume PBC, namely the positivity to AMA (a significant serologic marker) and an AP > 1.5 times the upper limit of normal for $>24$ weeks. But, from initial study, the patient had an elevation of AST ( $>2$ times the normal) and ALT (almost three times the normal) and a slight increase in serum IgG. Sometimes, the PBC can overlap with AIH. This overlap syndrome consists of a range of clinical entities of $\mathrm{AIH}$ and $\mathrm{PBC}$, with PBC generally preceding AIH by several years. The diagnosis of PBC and AIH overlap syndrome is made when presenting diagnostic criteria of PBC and criteria of AIH: 1) Serum alanine aminotransferase $\geq 5$ times the upper limit of normal, a positive test for ASMA or $\operatorname{IgG} \geq 2$ times the upper limit of normal; and 2) Liver biopsy demonstrating moderate or severe periportal or periseptal, lymphocytic and piecemeal necrosis [7]. In this case report, at this moment of disease evolution, we cannot assume with certain that this patient has a PBC and $\mathrm{AIH}$ overlap syndrome.

However, the histology of liver, with interface hepatitis and abundant inflammatory infiltrate, consisting predominantly of lymphocytes, is very suggestive of AIH. As the patient does not fulfill the criteria to AIH, we decide to perform the treatment with UDCA only, receiving the patient's good response to treatment, with normalization of AP, AST and ALT. Although IgM concentration does not form part of standard diagnostic criteria, an elevation of IgM is characteristic of PBC, which is useful in making a clinical diagnosis in patients with atypical other features. This patient had an elevation of IgM almost 3 times, and reduction after treatment with UDCA. IgM reduction after treatment has been reported, however the prognostic significance of it has yet to be established [11].

Freedman et al (2020), in a meta-analysis of treatment of overlap syndromes in autoimmune liver diseases, concluded that there were no clear differences in clinical outcomes between different treatment approach: UDCA only, immunosup- 
pression or combination of UDCA and immunosuppression, although the quality of evidence was low [13]. The patients with overlap syndrome may benefit from immunosuppressive treatment in addition to UDCA, with the immunosuppressive treatment recommended in patients with severe hepatitis. It should be considered in patients with moderate interface hepatitis [6].

In this case, we will still follow up the patient, looking for the onset of criteria of PBC and AIH overlap, until there, maintaining the UDCA treatment, but keeping alert to the need of corticoid association. For now, with the good response to UDCA, and pondering the potential benefit and risks of corticoid, we choose to wait and see.

\section{Acknowledgments}

None to declare.

\section{Financial Disclosure}

The authors have no conflict of interest to declare.

\section{Conflict of Interest}

None to declare.

\section{Informed Consent}

Informed consent was obtained from the patient.

\section{Author Contributions}

$\mathrm{VC}$ is the physician who worked with patient and contributed to manuscript design and writing; MM, RC and JF are the physicians who worked with patient and were involved in manuscript review; MM, RC and JF contributed to data collection and manuscript review.

\section{Data Availability}

The authors declare that data supporting the findings of this study are available within the article.

\section{References}

1. Gulamhusein AF, Hirschfield GM. Primary biliary cholangitis: pathogenesis and therapeutic opportunities. Nat Rev Gastroenterol Hepatol. 2020;17(2):93-110.

2. Lleo A, Jepsen P, Morenghi E, Carbone M, Moroni L, Battezzati PM, Podda M, et al. Evolving trends in female to male incidence and male mortality of primary biliary cholangitis. Sci Rep. 2016;6:25906.

3. Kaplan MM, Gershwin ME. Primary biliary cirrhosis. N Engl J Med. 2005;353(12):1261-1273.

4. Boonstra K, Beuers U, Ponsioen CY. Epidemiology of primary sclerosing cholangitis and primary biliary cirrhosis: a systematic review. J Hepatol. 2012;56(5):11811188.

5. Liberal R, Grant CR. Cirrhosis and autoimmune liver disease: Current understanding. World J Hepatol. 2016;8(28):1157-1168.

6. European Association for the Study of the Liver. Electronic address, easloffice easloffice eu, European Association for the Study of the Liver. EASL Clinical Practice Guidelines: The diagnosis and management of patients with primary biliary cholangitis. J Hepatol. 2017;67(1):145-172.

7. Purohit T, Cappell MS. Primary biliary cirrhosis: Pathophysiology, clinical presentation and therapy. World J Hepatol. 2015;7(7):926-941.

8. Gatselis NK, Dalekos GN. Molecular diagnostic testing for primary biliary cholangitis. Expert Rev Mol Diagn. 2016;16(9):1001-1010.

9. Czul F, Peyton A, Levy C. Primary biliary cirrhosis: therapeutic advances. Clin Liver Dis. 2013;17(2):229-242.

10. Bairy I, Berwal A, Seshadri S. Autoimmune hepatitis primary biliary cirrhosis overlap syndrome. J Clin Diagn Res. 2017;11(7):OD07-OD09.

11. Hirschfield GM, Dyson JK, Alexander GJM, Chapman $\mathrm{MH}$, Collier J, Hubscher S, Patanwala I, et al. The British Society of Gastroenterology/UK-PBC primary biliary cholangitis treatment and management guidelines. Gut. 2018;67(9):1568-1594.

12. Al-Harthy N, Kumagi T. Natural history and management of primary biliary cirrhosis. Hepat Med. 2012;4:61-71.

13. Freedman BL, Danford CJ, Patwardhan V, Bonder A. Treatment of overlap syndromes in autoimmune liver disease: a systematic review and meta-analysis. J Clin Med. 2020;9(5):1449. 